\title{
Radio resource coordination and scheduling scheme in ultra-dense cloud-based small cell networks
}

\author{
Zengxian Chen ${ }^{1 \dagger}$, Sai Zou ${ }^{1}$, Yuliang Tang ${ }^{1 *}+\mathbb{D}$, Xiaojiang Du² and Mohsen Guizani ${ }^{3}$
}

\begin{abstract}
In a 5 G ultra-dense network, dynamic network topology and traffic patterns lead to excessive system overhead and complex radio resource conflicts. The cloud radio access network and the fog computing have the advantages of high computation capabilities and low transmission delays. Therefore, by taking full advantage of these two characteristics, this study proposes a novel radio resource coordination and scheduling scheme in an ultra-dense cloud-based small cell network. Interference among small cells (or remote radio heads) can be avoided by implementing centralized cooperative processing in the base band unit pool in advance. Resource sharing in coordination and transfer depend on fog computing to relieve the overloaded cloud processing platform and reduce transmission delays, thereby maximizing resource utilization and minimizing system overhead when the network topology and number of users change dynamically. The simulation shows that the proposed scheme can increase the system throughput by $20 \%$ compared with the clustering-based algorithm; it can also increase system throughput by $33 \%$ compared with the graph coloring algorithm, decrease the signaling overhead by about 50\%, and improve network's quality of service.
\end{abstract}

Keywords: Ultra-dense network (UDN), The cloud radio access network (Cloud-RAN), Fog computing, Resource coordination, 5th generation (5G), Small cell

\section{Introduction}

Given current demand, broadband mobile data is expected to be ubiquitously available. The industry has predicted a 1000 -fold increase in mobile data traffic within the next decade. Ultra-dense network (UDN) deployment appears promising for improving network capacity $[1,2]$. Traditional operators must deploy more infrastructure to address myriad challenges associated with data proliferation, which increases total costs significantly [3, 4]. Radio resource management and scheduling in UDNs comprise an important means of network capacity promotion; however, because a larger number of small cells are needed to promote higher data capacity, problems related to interference and system overhead in the current UDN are much more severe than those in existing cellular mobile communication networks. The

\footnotetext{
*Correspondence: tyl@xmu.edu.cn

${ }^{\dagger}$ Equal contributors

${ }^{1}$ Department of Communication Engineering, Xiamen University, Xiamen 361005, China

Full list of author information is available at the end of the article
}

interference problem includes radio resource conflict and cell interference, whereas the system overhead problem affects delay, signaling interaction, and computational abilities.

The ultra-dense cloud-based small cell network combines cloud computing and fog computing in the development of a large number of small cells. The cloud radio access network (Cloud-RAN) presents a promising method for alleviating both capital and interference in UDNs, while providing high energy efficiency and capacity. Virtualized network technology is a key technology of resource management and scheduling in the fifth generation (5G) mobile communication networks [5, 6]. Virtualized network technology allows why Cloud-RAN to integrate centralization and virtualization into its architecture. The resources can be better managed and dynamically coordinated on demand on a pool level because Cloud-RAN centralizes all base band units (BBU) to form a pool, and remote radio heads (RRH) provide basic wireless signal coverage. In practice, the front haul and back haul of Cloud-RAN are often constrained by capacity and 
delays. Fog computing appears to be charged with most computation assignments and services from the cloud processing platform in the network's edge, which eases overload [7]. Clearly, Cloud-RAN with the better computational capability (10 times larger than that of the fog RAN) and the fog computing with a lower transmission delay are complementary in UDNs.

The main issue faced by resource management and scheduling is the reduction of inter-cell interference in 5G UDNs. Interference includes inter-small cell interference (ISI) and macro-small cell interference (MSI) [8]. MSI can be solved by using different frequencies; for instance, the macro cell uses a low frequency, and the small cell uses a high frequency [9]. The third generation partnership project (3GPP) R8 and R9 minimizes inter-cell interference in the macro and maximizes spectrum efficiency through frequency reuse planning. Due to the unplanned deployment of small cells, operators have little control over small cells' position and thus cannot devise frequency plans in advance. Therefore, the reasonable allocation of radio resources in small cells, such as frequency, has become the focus of current research to reduce the interference. Coordinated multi-point (CoMP) is a key method of mitigating inter-cell interference that can be applied to the network architecture, such as control/data plane separation architecture and Cloud-RAN [10].

Given growing demand for mobile data capacity, especially in urban areas, network densification is inevitable [11]. The user equipment in ultra-dense small cells has a tidal effect; the authors of [12] proposed a mobility-aware uplink interference model for $5 \mathrm{G}$ heterogeneous networks to solve the uplink interference with time variation. The authors of [13] presented a completely distributed channel allocation algorithm based on game theory. Although these methods improved radio resource utilization, they also created larger system overheads. To minimize ISI and ensure users' quality of service, some academics have used small cell clustering to coordinate cluster members' resource allocation. Considering graph theory, a clustering-based interference coordination heuristic algorithm was proposed in [14-16], which forms clusters dynamically and divides resource allocation into three phases to minimize ISI: (1) cluster formation; (2) intracluster resource allocation and admission control; and (3) inter-cluster resource contention resolution, in order to minimize ISI. It is worth noting that in this model, the formation of a given cluster is updated only when the interference caused by the changes in the user number exceeds a given threshold. In [17], authors devised a clustering and resource allocation for dense femtocells in a two-tier cellular orthogonal frequency division multiplexing access (OFDMA) network. This solution converts the interference problem into a mixed integer nonlinear optimization model, and the simulation results found this algorithm to be less complex. According to the clustered small cells and predicted signal to interference plus noise ratio (SINR), the authors of [18] proposed a power control scheme applied to the downlink in the small cell network and found this method to lower the likelihood of an outage while improving throughput significantly compared to previous methods. However, these methods require considerable signaling interaction between small cells to complete the resource allocation.

To adapt to the various demands of radio traffic, network virtualization and UDNs represent key technologies for future 5G wireless networks. In the virtualization architecture of $5 \mathrm{G}$ wireless networks, significant changes occur in the signaling interaction of virtual cells and the management mode of multi-dimension wireless networks. Based on interference coordination in traditional cells, the current network needs a more flexible and efficient sharing of radio resources to realize their coordinated allocation, including in the time, frequency, spatial, and power domains $[19,20]$. The wireless network virtualization technology forms a virtual network in isolation for different users by sharing the network's physical infrastructure and radio resources to achieve efficient use in $[5,6]$. A centralized resource pool must be built to achieve dynamic sharing and coordination. The resource pool provides the distributed function unit a logically centralized radio resource and processing resource to decouple resource and network functions, to realize resource sharing and distribution in different network entities, and to improve resource utilization overall [21, 22]. In [23, 24], a study conducted on interference coordination in a heterogeneous wireless virtualization network, using power control to reduce interference between small cells and thereby improving coverage and indoor small cells' capacity.

However, the interference and system overhead remains more severe in 5G UDNs due to dynamic traffic and the random increase or migration of small cells. Current resource allocation algorithms for small cells are unable to manage poor situations, an urgent problem that requires an innovative solution. Inspired by the method of collaboration between small cells in COMP, a resource coordination approach was proposed in [25] to solve the problem. Considering Cloud-RAN and fog computing, this study presents a novel resource coordination and scheduling scheme in ultra-dense cloud-based small cells.

The rest of this paper is organized as follows. Section 2 introduces the methods of this paper. Section 3 describes the system model of Cloud-RAN and fog computing. Section 4 details the resource coordination and scheduling scheme in ultra-dense small cells. Section 5 describes a resource scheduling example and corresponding analyses of signaling overheads. Scenario setting of simulation are presented in Section 6, and the results and discussion 
of this paper are proposed in Section 7. Finally, the conclusion and ending remarks are given in Section 8.

\section{Methods}

Considering Cloud-RAN and fog computing, this study presents a novel resource coordination and scheduling scheme in ultra-dense cloud-based small cells to improve system throughput and reduce system signaling overhead. After network initialization is accomplished, a global resource allocation algorithm is used in the cloud processing platform to allocate resources for each fog device according to the global signaling request. When the tidal effect occurred or resources are deficient, the fog devices in the edge of the cloud executes the partial resource optimization algorithm or resource coordination and scheduling scheme to adjust the number of resources between each fog device. Resource coordination between fog devices can be divided into three situations: (1) when some small cells power off, the cloud-based system will obtain resource of the fog device that these small cells belong to; (2) when some small cells power on, the cloud adjusts the resources between the fog device, whose small cells power on, and its adjacent fog device to ensure a minimal amount of physical resource block (PRB) switching in subsequent processes; (3) when fog device resources are insufficient, the fog device lacking resources needs to either send a request for resources to the system resource pool in the cloud or borrow resources from adjacent fog devices, a process that also aims to minimize the amount of PRB switching.

\section{System model}

Multiple small cells are deployed in the area with a density of 177 cells per $\mathrm{km}^{2}$, and any two cells whose signals interfere with each other are termed "adjacent cell." This study uses the differ-frequency development scenarios of macro-small cell 3GPP TR 36.932 proposed; there is no co-channel interference between the macro cell and small cells due to the differ-frequency network. Based on the architecture of Cloud-RAN and fog computing, small cells are spliced as BBU and RRH, and the fog device connects the cloud processing platform via the back haul network and connects the corresponding small cells via front haul network. As shown in Fig. 1, a BBU pool is located in a centralized cloud processing platform that is used to manage the radio resources in the system resource pool. The cloud processing platform manages all fog devices, and different fog devices manage small cells in various traffic areas. There may be more than two fog devices in system; small cells with the same characteristic (depending

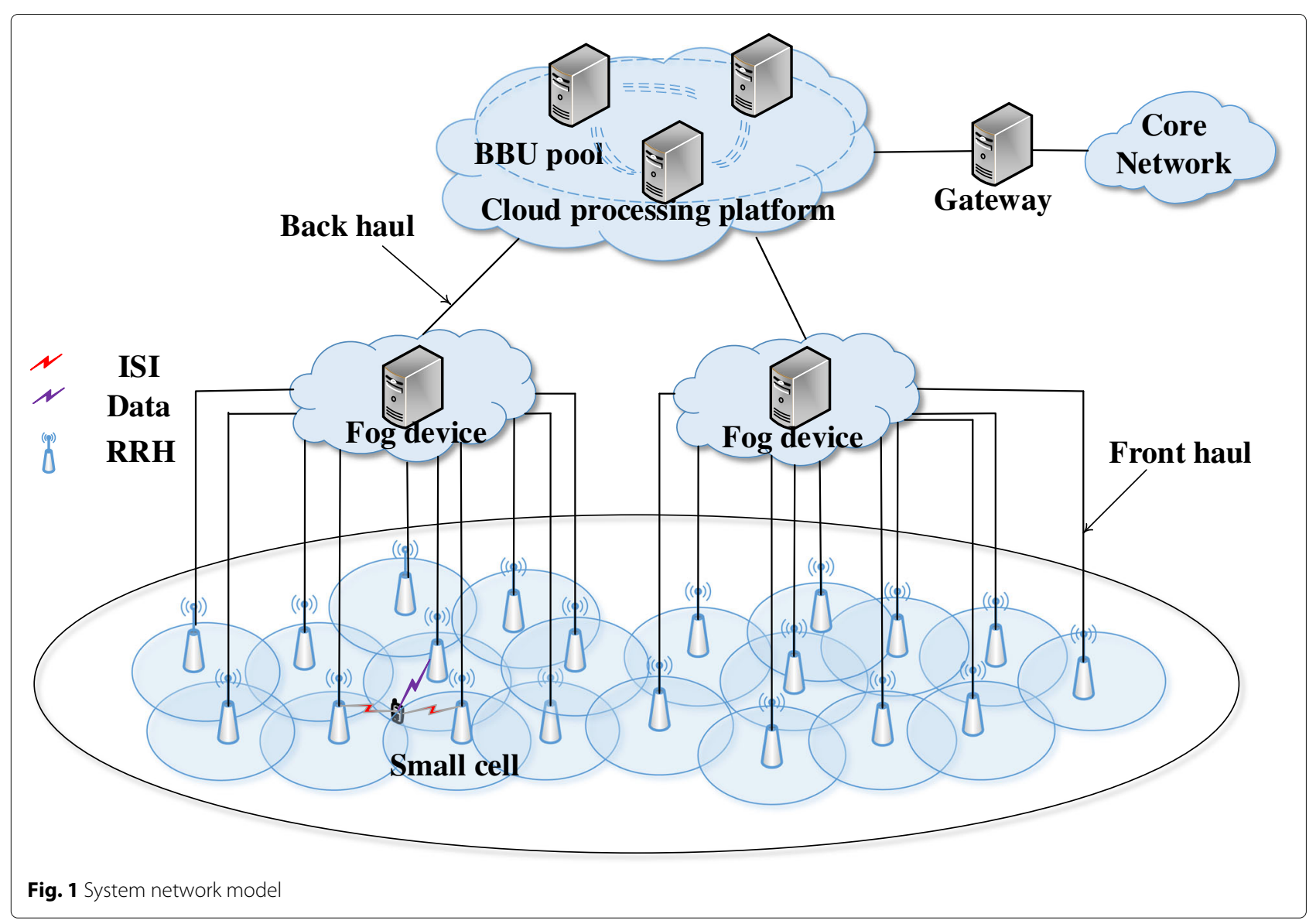


on traffic and location) connect to the associated fog device via a front haul network. Different fog devices can communicate when resource coordination is required.

A table of notations for this paper is provided in Table 2. Set $\mathcal{S}=\left\{s_{1}, \ldots, s_{m}\right\}$ as the set of small cells in the area $G$; $m \in \mathbb{N}^{*}, \mathbb{N}^{*}$ represents the set of positive integers. $\mathcal{B}=$ $\left\{b_{1}, \ldots, b_{n}\right\}$ donates PRBs in the system resource pool, and $n \in \mathbb{N}^{*} . \mathcal{U}=\left\{u_{1}, \ldots, u_{o}\right\}$ is a set of user equipment (UE) in a small cell, $o \in \mathbb{N}^{*}$. Due to the downlink interference, the rate $R_{i, k, j} \mathrm{UE} u_{k}$ obtained based on PRB $b_{j}$ in small cell $s_{i}$ is

$$
R_{i, k, j}=W \cdot \log _{2}\left(1+\operatorname{Sin} r_{i, k, j}\right),
$$

where $W$ is the bandwidth of PRB, and $\operatorname{Sin} r_{i, k, j}$ represents the SINR UE $u_{k}$ received based on PRB $b_{j}$ in $s_{i}$, which can be calculated by

$$
\operatorname{Sin} r_{i, k, j}=\frac{p_{i, k, j} \cdot g_{i, k, j}}{\sum_{\substack{l=1 \\ l \neq i}}^{m} p_{l, k, j} \cdot g_{l, k, j}+n_{0}},
$$

where $p_{i, k, j}$ indicates the transmission power from serving small cell $s_{i}$ to UE $u_{k}$ based on PRB $b_{j}, p_{l, k, j}$ indicates the interference power from intruder small cell $s_{l}$ to UE $u_{k}$ based on PRB $b_{j}, n_{0}$ stands for the noise power, and $g_{i, k, j}$ is the channel gain, and the channel gain is composed of link loss and the antenna gain.

Set $\mathcal{B S} \mathcal{S}_{i}=\left\{b_{1} s_{i}, b_{2} s_{i}, \ldots\right\}$ as the available PRB set of small cell $s_{i}$. For any UE $u_{k}$ in the network, $R_{k}$ is a constant used to indicate the minimum rate demand of UE $u_{k}$. The constraint that the UE $u_{k}$ can access the small cell $s_{i}$ at a certain position is

$$
\sum_{b_{j} s_{i} \in \mathcal{B} \mathcal{S}_{i}} R_{i, k, j} \geqslant R_{k}
$$

where the summation $\sum_{b_{j} s_{i} \in \mathcal{B S}_{i}} R_{i, k, j}$ stands for the current rate that the UE $u_{k}$ obtained when all the PRBs in small cells $s_{i}$ are allocated to the UE $u_{k}$, which must be greater than or equal to the minimum rate demand $R_{k}$ of the UE $u_{k}$.

\section{Resource coordination and scheduling scheme in small cells}

The problem of resource allocation in this paper is similar to graph coloring problem (GCP). The same PRB cannot be assigned to adjacent nodes no matter which node the resource allocation starts from, until the PRB resource allocation of all nodes has completed. Moreover, the problems in our paper are different from GCP. Each node has only one color in GCP, but each nodes needs to be allocated one or more PRBs in our problems, so it not only belongs to GCP but also belongs to subset selection problem. GCP is one of $33 \mathrm{NP}$-hard problems, and subset selection problem has been proven to be NP-hard problem in [26]. Therefore, heuristic algorithm is needed to solve the problem of resource allocation in this paper. A novel radio resource coordination and scheduling scheme in small cells (including three heuristic algorithms) are proposed to solve the resource allocation problem. A specific function diagram of the scheme is shown in Fig. 2. Global resource allocation and partial resource allocation are executed in a cloud processing platform with increased computational capabilities. The resource coordination and scheduling is processed in a fog device with larger storage. First, the cloud processing platform allocates PRB for each fog device to try to meet the pre-demands of small cells using minimum PRB in the system resource pool, thereby reducing the signaling overheads and ensuring interference optimization in the whole network. Then, the cloud processing platform updates real-time interference graphs (mentioned in Section 3.1.2) with topology changes, and a partial resource allocation algorithm is used to adjust the number of allocated PRBs in each fog device. A resource request



Fig. 2 Function block diagram of the proposed scheme 
to the fog device will be sent as UE is added or switched in a small cell if resources are lacking. If there are no available idle resources in the system resource pool or in adjacent small cells, then a PRB transfer process will be launched for the small cell. The adjacent small cells transfer their PRBs to the small cell following certain rules, and the rate compensation is executed in adjacent small cells; that is, the fog device finds available PRBs for the small cells with rate loss. This process aims to achieve minimal PRB switching while facilitating user access.

\subsection{Global resource allocation algorithm}

\subsubsection{Global resource allocation model}

Based on the system model in Fig. 1, the cloud processing platform allocates a certain number of PRBs for each fog device, and the fog device has a PRB list of small cells. This process avoids interference to reduce user access delay and signaling overheads. The problem of global resource allocation can be transformed into an optimization model, which is aimed at the maximum number of remainder PRBs in the system resource pool after each allocation, as shown in formula (4).

$$
\arg \max \sum_{i=1}^{m}\left(n_{i}^{\prime}-Y_{i}\right)
$$

subject to

$$
\begin{aligned}
& Y_{i}=\left\lfloor\frac{n}{1+\sum_{\substack{l=1 \\
l \neq i}}^{m} I_{i, l}}\right\rfloor_{\substack{l \neq i \\
b_{i}}}(4 b) \\
& \forall b_{j} \in \mathcal{B S}_{i} I_{i, l}=1, \exists b_{j} \notin \mathcal{B S}_{l},(4 c)
\end{aligned}
$$

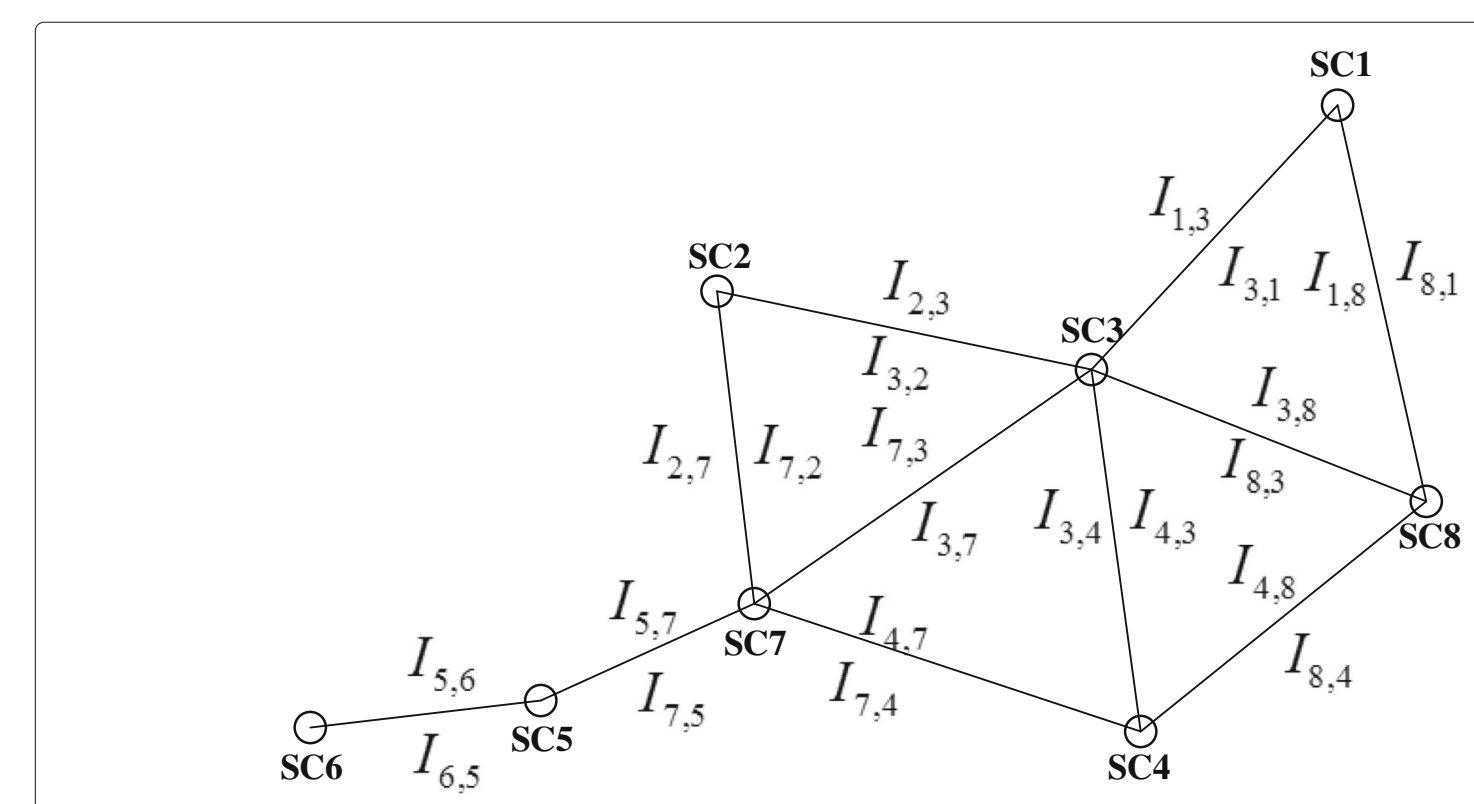

Fig. 3 The global interference graph where $n$ represents the total number of PRBs in the system resource pool in its initial state, $n^{\prime}$ denotes the total number of PRBs in the system resource pool before each allocation, $n_{i}^{\prime} \leqslant n$, and $n_{i}^{\prime}-Y_{i}$ represents the remaining PRBs in the system resource pool after allocating for small cell $s_{i} . I$ is an interference matrix of small cells. Any two cells whose signals interfere with each other are termed "adjacent cells." If $s_{i}$ and $s_{l}$ are adjacent cells, according to the interference weight, $I_{i, l}, I_{l, i} \in(0,1]$; otherwise, $I_{i, l}=$ $I_{l, i}=0$. A kind of demand function $Y_{i}$ is proposed and shown in formula $(4 b)$ for interference optimization and fairness in the whole network. $Y_{i}$ means that the total PRB in the system resource pool is shared equally by a small cell and its adjacent cells. In other words, the more adjacent cells a small cell has, the more interference it causes, and the $Y_{i}$ is smaller. $\lfloor *\rfloor$ represents rounding down to the nearest integer. The constraint $(4 c)$ means that two small cells with interference cannot be allocated to the same PRB.

\subsubsection{Solution of global resource allocation model}

In this section, a global resource allocation optimization algorithm is proposed to solve the above optimization model. The algorithm is as follows:

1. Each small cell scans the field strength and forms an interference diagram of its neighbor cells. Meanwhile, the small cell reports the interference diagram to the cloud processing platform.

2. The cloud processing platform generates the global interference graph shown in Fig. 3 and forms the interference matrix. The vertex $V$ of 
the interference graph represents the small cell in area $G$. The edge $E$ of the interference graph stands for the conflicting relationship between any two small cells that need to be avoided in interference. $I_{i, l}$ and $I_{l, i}$ indicates the weight of edge connecting small cells $s_{i}$ and $s_{l} . I_{i, l}$ depends on the SINR level in small cell $s_{i}$ considering the interference of small cells $s_{l}$, and $I_{l, i}$ depends on the SINR level in small cell $s_{l}$ considering the interference of small cell $s_{i}$. In other words, the larger SINR is, the smaller the corresponding interference weight is. Here, we normalized the interference weights, $I_{i, l}, I_{l, i} \in[0,1]$. Any two vertexes with an edge cannot be allocated to the same PRB.

3. According to the formula (4b), the cloud processing platform calculates the number of allocated PRBs for each small cell in the set of $\mathcal{S}^{\prime}$.

4. The cloud processing platform allocates PRBs for small cell $s_{i}^{\prime}$ and chooses the PRB with the smallest serial number in the system resource pool until the PRB number reaches $Y_{i}$, and the chosen PRB has not been allocated to its adjacent cells.

The pseudo code of algorithm 1 is as follows:



\subsection{Partial resource optimization algorithm}

When the network topology changes, a partial resource optimization algorithm is proposed, which is used to reduce amount of PRB switching caused by twice global allocation, thus improving user satisfaction. When the number of small cells in the network declines, such as when a small cell powers off, the system resource pool will recover the PRB from the used PRB list of the powered-off small cell. If the network adds small cell $s_{i}$, the mathematical optimization model aimed at the minimum amount of PRB switching is

$$
\arg \min \sum_{i=1}^{m} T_{i}
$$

subject to

$$
\begin{aligned}
& \operatorname{size}\left(\mathcal{B S}_{i}^{\prime}\right) \propto Y_{i} . \\
& \operatorname{size}\left(\mathcal{B}^{\prime \prime}\right) \propto \operatorname{size}\left(\mathcal{B}^{\prime}\right) .
\end{aligned}
$$

Here, the amount of PRB switching is defined as the sum of the number of PRB changes used by the cell after optimization. $T_{i}$ stands for the extent of PRB switching caused by small cell $s_{i}$. $\operatorname{size}\left(\mathcal{B S}_{i}^{\prime}\right)$ represents the total number of allocated PRBs in small cells after global allocation. $\operatorname{size}\left(\mathcal{B}_{i}^{\prime}\right)$ indicates the total number of PRBs in the system resource pool before partial resource optimization, while $\operatorname{size}\left(\mathcal{B}_{i}^{\prime \prime}\right)$ is the total number of PRBs in the system resource pool thereafter. The constraint $(5 b)$ indicates that the total number of practically allocated PRBs for small cell $s_{i}$ is equal to or near the number of theoretically allocated PRBs for small cell $s_{i}$. The constraint (5c) shows that the number of remaining PRBs in the system resource pool is unchanged after resource optimization.

To solve problem (5), the specific process of the resource allocation algorithm is as follows:

1. A new small cell scans the field strength and formats an interference diagram of neighbor cells. Meanwhile, the new small cell reports the interference diagram to the cloud processing platform.

2. The cloud processing platform updates the interference graph of adjacent cells according to the report on the relationship of the new small cell's neighbors.

3. The cloud processing platform calculates the number of allocated PRB for new small cell using formula $(4 b)$.

4. $\mathcal{B Z}_{s_{i}}$ stands for the idle PRB list belonging to

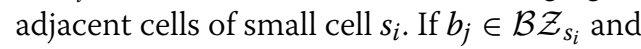
$b_{j} \notin \mathcal{B}^{\prime}$, the cloud processing platform allocates PRB $b_{j}$ to small cell $s_{i}$.

5. If there are more than two adjacent cells with idle allocated PRB, the cloud processing platform adjusts the PRB allocation of adjacent cells according to fairness principles and tries its best to meet the demand of PRB allocation for small cell $s_{i}$. Meanwhile, the cloud processing platform deletes the corresponding PRB allocated for small cell $s_{i}$ from the idle PRB list of adjacent cells. 
The fairness principles are as follows: set $P\left(C_{i}\right)$ to be the probability that PRB $b_{j}$ is capable of being borrowed, and set $P\left(D_{i}\right)$ to be the probability that PRB switching is generated in adjacent cells after borrowing PRB $b_{j}$. There is an adverse effect on subsequent resource allocation after borrowing some certain PRBs; thus, the cloud processing platform chooses the PRB with the smallest posterior probability $P\left(C_{i} \mid D_{i}\right)$ to allocate to the new small cell.

Because $P\left(C_{i} \mid D i\right)=\frac{P\left(D i \mid C_{i}\right)}{P\left(D_{i}\right)}$ and $P\left(D_{i}\right)$ does not depend on $C_{i}$, the cloud processing platform selects the PRB with the smallest posterior probability to transfer to small cell $s_{i}$ is shown in formula (6).

$$
\log P\left(C_{i}, D_{i}\right)=\log \left(P\left(D_{i} \mid C_{i}\right)\right)+\log P\left(C_{i}\right) .
$$

$\log P\left(C_{i}, D_{i}\right)$ is a type of Bayesian score. The prior distribution for parameter $\theta_{c}$ is $P\left(\theta_{c} \mid C_{i}\right) . P\left(D_{i} \mid C_{i}\right)$ in the formula (6) is expanded to get the formula (7).

$$
P\left(D_{i} \mid C_{i}\right)=\int_{-\infty}^{+\infty} P\left(D_{i} \mid C_{i}, \theta_{c}\right) \cdot P\left(\theta_{c} \mid C_{i}\right) d \theta_{c} .
$$

The first term of the integral in the formula (7) is deconstructed to obtain the next formula.

$$
\begin{aligned}
P\left(D_{i} \mid C_{i}, \theta_{c}\right)= & \prod_{i} \prod_{j} \prod_{k}\left(\theta_{i, j, k}^{\circ}\right)^{N_{i, j, k}^{\circ} .} \\
& \prod_{i} \prod_{j} \prod_{k}\left(\theta_{i, j, k}^{\prime}\right)^{N_{i, j, k}^{\prime}},
\end{aligned}
$$

where $\theta_{i, j, k}=P\left(x_{i}^{k} \mid a_{i}^{j}\right)$ is the conditional probability where $x$ is equal to $k$ in the case such that the parent node of the $i$ th node $x$ is $j$. Therefore, the prior distribution for parameter $\theta_{c}$ can be broken down into the following formula:

$$
P\left(\theta_{c} \mid C_{i}\right)=\prod_{i} \prod_{j} P\left(\theta_{i, j, k}^{\circ}\right) \cdot \prod_{i} \prod_{j} P\left(\theta_{i, j, k}^{\prime}\right) .
$$

Apply the formula (8) and (9) to the formula (7).

$$
P\left(D_{i} \mid C_{i}\right)=\prod_{i} \prod_{j} \int_{-\infty}^{+\infty} \prod_{k}\left(\theta_{i, j, k}^{\circ}\right)^{N_{i, j, k}^{\circ}} \cdot P\left(\theta_{i, j, k}^{\circ}\right) d \theta_{i, j, k}^{\circ}
$$

Assuming that the initial variables $N_{i, j, k}^{\circ}$ and the transfer variables $N_{i, j, k}^{\prime}$ have been selected and the observed data are complete, the problem can be solved using a heuristic algorithm (e.g., the greedy algorithm, genetic algorithm, tabu search algorithm, or ant colony algorithm).

\subsection{Resource coordination and scheduling algorithm}

In the system, each small cell has an idle allocated PRB list and a used PRB list. When a new UE or switching UE initiates an access request to the serving small cell, the small cell needs to get the reusable PRB if its idle PRB is unable to satisfy the rate demand of UE. This process may cause PRB switching in adjacent cells. In order to guarantee minimal PRB switching in the whole network, and to ensure the network throughput does not drop, a mathematical optimization model is depicted as (11).

$$
\begin{gathered}
\arg \min \sum_{i=1}^{m} T_{i} \\
\text { subject to } O_{\text {downstream }}^{\prime}>O_{\text {downstream. }} .
\end{gathered}
$$

The system downlink throughput $O_{\text {downstream }}$ in certain transmission time $t$ is calculated as

$$
O_{\text {downstream }}=\sum_{u_{k \in \mathcal{U}}} R_{k} \cdot \frac{\left(t-t_{\text {delay }}\right)}{t},
$$

where $t_{\text {delay }}$ is a certain constant. $O_{\text {downstream }}$ represents the system downlink throughput before each resource coordination, while $O_{\text {downstream }}^{\prime}$ represents the system downlink throughput after each resource coordination. The constraint $(9 b)$ means that the system downlink throughput will be increased after resource coordination.

To solve problem (11), a small cell resource coordination and scheduling algorithm (RCS) is proposed in fog device. The specific implementation process of RCS algorithm is as follows:

1. The fog device counts the small cells with new PRB demands for the formation of the set $\mathcal{S}^{\prime \prime}=\left\{s_{1}^{\prime \prime}, s_{2}^{\prime \prime}, \ldots\right\}$.

2. If the remaining PRB of the system resource pool can meet the new PRB demands, the fog device allocates the PRB to small cell $s_{i}^{\prime \prime}$ and deletes the PRB from the system resource pool. Then, the process jumps to step 7 , where the resource pool will recover the PRB when the user communication is completed; otherwise, the fog device solves unmet PRB demands of small cell $s_{i}^{\prime \prime}$ in the next step.

3. If the adjacent cells have idle PRBs, the fog device distributes these PRBs to the small cell $s_{i}^{\prime \prime}$. If the PRB requirements have been met, the process proceeds to step 7 . Otherwise, the process proceeds to step 4 .

4. If small cell $s_{i}^{\prime \prime}$ never initiates the PRB transfer process but the transfer will improve the system throughput, the fog device counts the PRB adjacent cells used and calculates the PRB value using the formula shown in (13). The PRB with the maximum value is prohibited from transferring. The fog device selects the PRB with the minimum value to transfer to meet the demand of small cell $s_{i}^{\prime \prime}$. In particular, if multiple PRBs have equal values, the fog device chooses the PRB with the smaller index to transfer; otherwise, the process proceeds to step 7 . 


$$
V_{j}=\prod_{s_{l} \in \mathcal{Z}_{s_{i}^{\prime \prime}}} \frac{w_{j, l}}{\operatorname{size}\left(\mathcal{B S}_{l}^{\prime \prime}\right)} .
$$

Here, $V_{j}$ stands for the value of PRB $b_{j}$ in adjacent cell $s_{l}$, and $\mathcal{Z}_{s_{i}^{\prime \prime}}$ represents the adjacent cell set of small cell $s_{i}^{\prime \prime}$. The fog device forms the PRBs set $\mathcal{B S}_{l}^{\prime \prime}$ by sorting the PRB in ascending order based on the use value in adjacent cells. PRB's use value depends on the actual rate obtained by UE who allocated this PRB in small cell. $w_{j, l}$ stands for the index of PRB $b_{j}$ in $\mathcal{B S}_{l}^{\prime \prime}$.

5. If the PRB small cell $s_{i}^{\prime \prime}$ needs have been met, the small cell $s_{i}^{\prime \prime}$ exits the resource scheduling process and does not participate in subsequent coordination, the process proceeds to step 6. Otherwise, the process proceeds to step 7 .

6. The fog device calculates the rate loss of a small cell after transfer based on Eq. (14) and sorts small cells in descending order according to the rate loss. The ordered results will be sorted into the set $\mathcal{S}^{\prime \prime}$, prioritizing the resource scheduling process to those small cells whose new PRB demands have not yet been met.

$$
L_{l}=\frac{\sum_{j=1}^{b_{j}{ }^{\prime \prime} \in \mathcal{T} \mathcal{S}_{l}} R_{l, k, j}}{\sum_{j=1}^{b_{j} s_{l}^{\prime \prime} \in \mathcal{B} \mathcal{S}_{l}^{\prime \prime}} R_{l, k, j}}
$$

In this equation, $L_{l}$ represents the rate loss of adjacent cell $s_{l}, \mathcal{T} \mathcal{S}_{l}$ denotes the set of transferred PRBs in adjacent cell $s_{l}$, and $\mathcal{B S}_{l}^{\prime \prime}$ stands for the PRB list of adjacent cell $s_{l}$ before the transfer.

7. If the PRB demands in the set $\mathcal{S}^{\prime \prime}$ have been solved, the algorithm ends; otherwise, the process proceeds to step 2 .

\section{Resource scheduling example}

This section introduces a PRB scheduling example of small cells to better explain the implementation process of the proposed scheme. Small cells belong to the same fog device as they are deployed in the adjacent office of the same building. Seven small cells $s_{1}, s_{2}, \ldots, s_{7}$ power on, while $s_{8}$ is in a shutdown state. After a certain period of time, $s_{8}$ powers on and can no longer accommodate a new access request. In this situation, a system resource allocation conflict occurs. As is shown in Fig. 4, in actual network operation, there is an example of resource scheduling in managed area of a fog device. In this example, the "j" stands for $b_{j}$ having been used; " $j$ " indicates PRB $b_{j}$ has not been used. The scheduling case is primarily divided into four processes:
1. The cloud processing platform allocates PRB for the powered-on small cells based on the global resource allocation algorithm.

2. After small cell $s_{8}$ powers on, the fog device borrows PRB $b_{3}$ and $b_{4}$ from the idle PRB list in adjacent cells to allocate for small cell $s_{8}$ based on the partial resource optimization algorithm. In this case, $b_{9}$ and $b_{10}$ remain in system resource pool.

3. Small cell $s_{8}$ has five new PRB demands and uses the allocated $b_{3}$ and $b_{4}$. The new PRB demand of $s_{8}$ has not been met. At this time, a system resource allocation conflict occurs. The fog device finds available PRBs using a resource coordination and scheduling algorithm. The specific steps of this process are as follows:

(a) The fog device allocates PRB $b_{9}$ and $b_{10}$ in the system resource pool to small cell $s_{8}$ and deletes the corresponding PRB from the resource pool. The new PRB demand of small cell $s_{8}$ has not been satisfied, and adjacent cells have no idle PRB. The fog device launches the transfer process for small cell $s_{8}$ and uses the formula (13) to calculate the value of PRB used in adjacent cells. The PRB with the maximum value is prohibited from transferring. The fog device selects the PRB $b_{5}$ with the minimum value to transfer to small cell $s_{8}$. The PRB demands of small cell $s_{8}$ have been met, and small cell $s_{8}$ exits the resource scheduling process and does not participate in subsequent coordination. The rate loss caused by the transfer of small cell $s_{1}$ is $\frac{1}{2}$, and that caused by the transfer of small cell $s_{4}$ is $\frac{1}{3}$.

(b) The fog device launches the rate compensation for small cell $s_{1}$, and small cell $s_{1}$ uses its own allocated PRB $b_{7}$ to meet demands. Then, the fog device initiates the rate compensation for small cell $s_{4}$ and allocates the unused PRB $b_{8}$ in adjacent cells to small cell $s_{4}$. Once the resource requirements of all small cells in the system have been met, the algorithm ends.

Assume there is no UE access to small cell $s_{8}$ for an extended period of time, and the allocated PRBs of small cell $s_{8}$ are all borrowed to its adjacent cells. Conversely, when there are new accessed users in small cell $s_{8}$, the system will initiate the PRB transfer process. As shown in Fig. 5, there are two new PRB demands in the poweredon small cell $s_{8}$. In order to allocate PRB for new users of small cell $s_{8}$, the PRB transfer will occur among the other small cells. As a result, small cell $s_{8}$ will get enough 


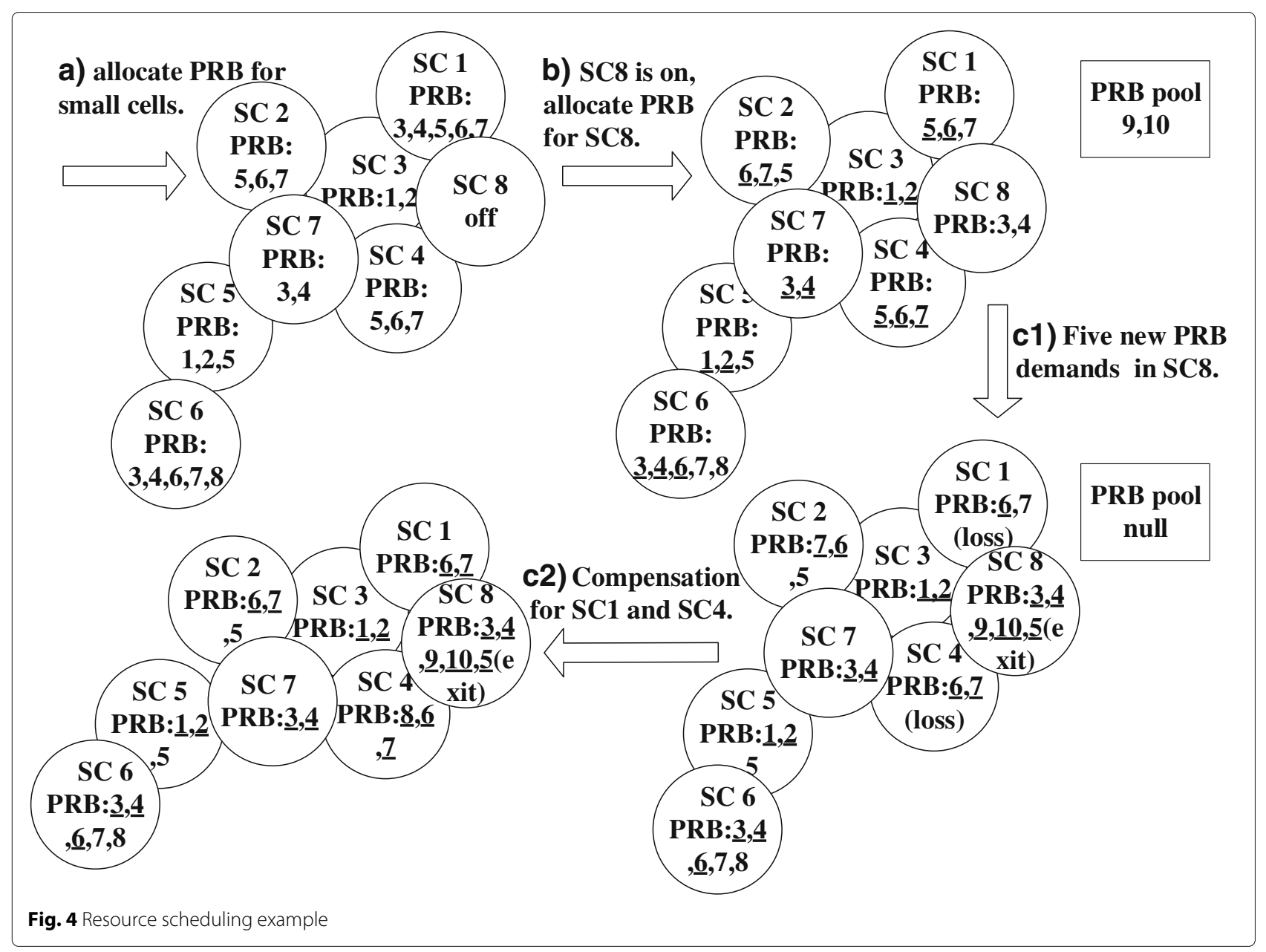

PRB for its new users, while the used PRB from other small cells will need to make additional PRB switches. The system overhead and transmission delay caused by PRB switching should be minimized as much as possible. Here, we give a scheduling case comparison of the clusteringbased interference coordination algorithm [16] and the graph coloring algorithm [27] to our algorithm based on the allocation in Fig. 5 to analyze and contrast transmission delay and signaling overheads. The clustering-based interference coordination algorithm was described in the introduction, and the graph coloring algorithm is a type of greedy algorithm. The latter is a heuristic method of solving the inter-cell interference problem and involves two main stages: (i) the formation of interference graph and (ii) the resource allocation solution. The resource allocation solution means that each nodes must be allocated a preset number of colors, and no two adjacent nodes may have any colors in common. The objective is to complete this using the fewest possible number of colors. PRB switching occurs three times in the proposed algorithm, six times in the clustering-based algorithm, and nine times in the graph coloring algorithm. The global resource allocation can reduce the delay of PRB allocation for new user access. In this case, small cell $s_{8}$ is the worst case, and it does not fully leverage the advantages of allocation. However, the algorithm still reduces the amount of PRB switching caused by resource conflicts and lowers the system overheads.

\section{Simulation}

\subsection{Scenario setting}

In the simulation scenario, 64 small cells were deployed within a macro cell coverage range. The macro and small cells used differ-frequency. UE randomly arrived at a small cell at the arrival rate of $30 \mathrm{user} / \mathrm{s}$, and its transmission rate demands range from 1 to $1000 \mathrm{~kb} / \mathrm{s}$. There were 25 total PRBs in the system. The specific simulation parameter will be written as shown in Tables 1 and 2 . To determine the performance of the proposed algorithm, we compared it to two other algorithms with regard to the following: system throughput, average user SINR, user satisfaction, and signaling overheads. 


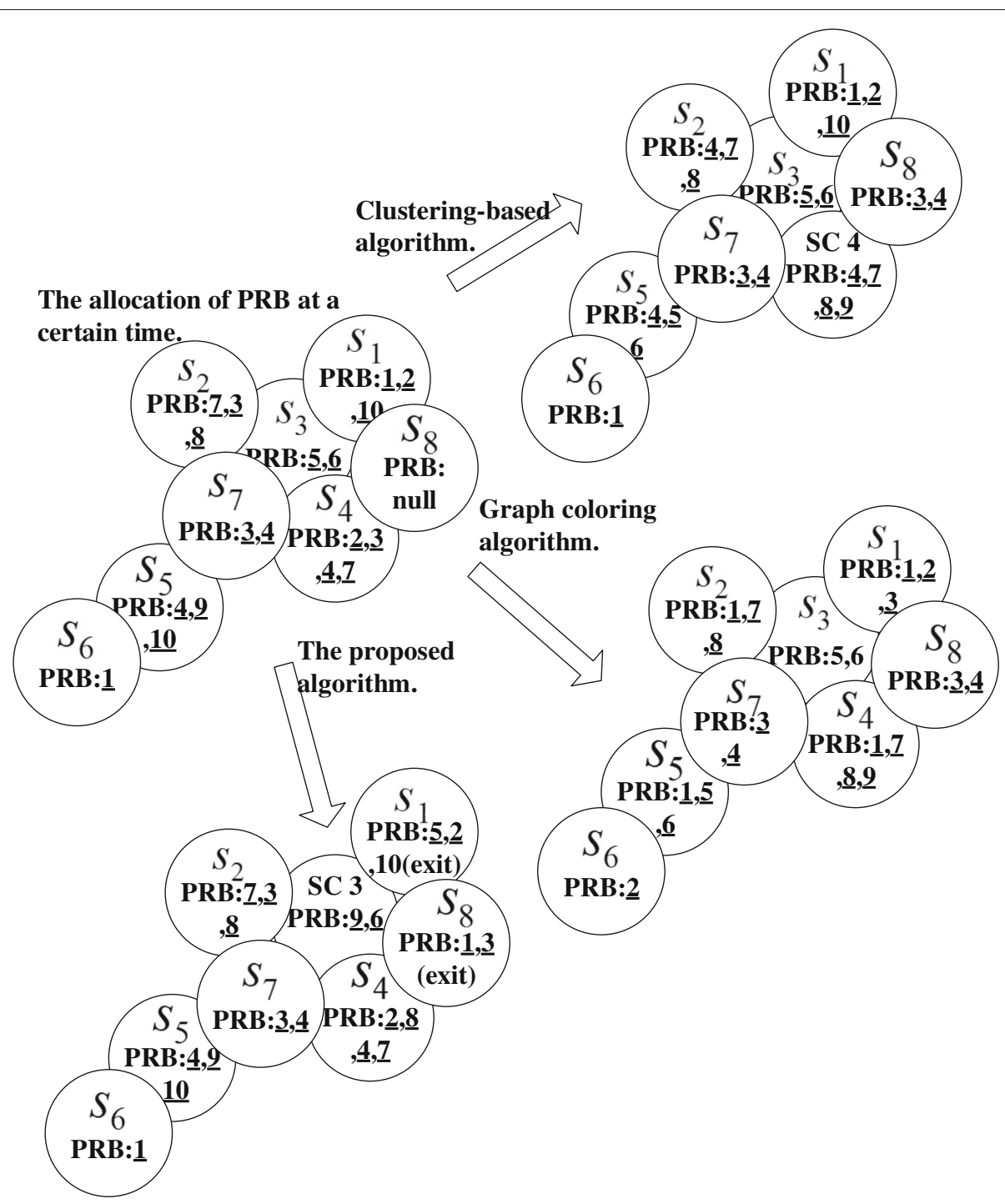

Fig. 5 Comparison scheduling case with other two algorithms

Table 1 System parameter

\begin{tabular}{ll}
\hline Parameters & Value \\
\hline Simulation area size & $600 \mathrm{~m}^{*} 600 \mathrm{~m}$ \\
Bandwidth of network & $5 \mathrm{MHz} / 2 \mathrm{GHz}$ \\
Antenna gain of small cell & $5 \mathrm{~dB}$ \\
Maximum transmit power of BS & $24 \mathrm{dBm}$ \\
Radius of the small cell & $40 \mathrm{~m}$ \\
Maximum user transmit power & $23 \mathrm{dBm}$ \\
Handover delay & $20 \mathrm{~ms}$ \\
White noise & $-174 \mathrm{dBm} / \mathrm{Hz}$ \\
Shadow fading standard deviation & $3 \mathrm{~dB}$ \\
Number of User[initial value: step: final value] & {$[30: 30: 750]$} \\
Buffer model & Full buffer \\
\hline
\end{tabular}

\section{Results and discussion}

\subsection{Analysis of throughput and SINR}

The simulation curves in this study are zigzagged because the user arrival rate (30 user/s) caused the performance index to change significantly. As shown in Fig. 6, when the user number is less than 210 , the system resources are abundant and can meet users' demands, and the system throughput increases overall. The system throughput reaches a maximum about $121 \mathrm{Mbps}$ when the user number is equal to 210 . The proposed algorithm satisfied the resource demands of users in serving small cells by borrowing resources from adjacent cells. Some users were assigned the PRB with a low rate and high reusability, which scarified the benefit for users assigned this $\mathrm{PRB}$, and clearly improved the throughput of a new user obtaining this PRB; therefore, the system throughput is improved significantly. When the user number gradually 
Table 2 Table of notations

\begin{tabular}{|c|c|}
\hline Symbol & Meaning \\
\hline $\mathcal{S}=\left\{s_{1}, \ldots, s_{m}\right\}$ & $\begin{array}{l}\text { Set of small cells in the whole network, } m \in \mathbb{N}^{*} \text {, and } \\
\mathbb{N}^{*} \text { represents the set of positive integers. }\end{array}$ \\
\hline $\mathcal{B}=\left\{b_{1}, \ldots b_{n}\right\}$ & Set of PRBs in the system resource pool, $n \in \mathbb{N}^{*}$. \\
\hline $\mathcal{U}=\left\{u_{1}, \ldots, u_{0}\right\}$ & Set of users (UE) of a small cell, $o \in \mathbb{N}^{*}$. \\
\hline$R_{i j, k}$ & $\begin{array}{l}\text { The rate user } u_{k} \text { obtained based on PRB } b_{j} \text { in small } \\
\text { cell } s_{i} \text {. }\end{array}$ \\
\hline W & Bandwidth of per PRBs. \\
\hline Sinr $r_{i, j, k}$ & SINR $u_{k}$ received based on PRBs $b_{j}$ in small cell $s_{i}$ \\
\hline$g_{i j, k}$ & Channel gain. \\
\hline p & Transmitted power of small cell. \\
\hline$n_{0}$ & Noise power. \\
\hline \multicolumn{2}{|c|}{$\mathcal{B S}_{i}=\left\{b_{1} s_{i}, b_{2} s_{i}, \ldots,\right\}$ The set of available PRBs in small cell $s_{i}$} \\
\hline$R_{k}$ & A constant to indicate the rate demand of user $u_{k}$. \\
\hline$n$ & $\begin{array}{l}\text { Total number of PRB in the system resource pool in } \\
\text { initial state. }\end{array}$ \\
\hline$n_{i}^{\prime}$ & $\begin{array}{l}\text { Total number of PRBs in the system resource pool } \\
\text { before each pre-allocation, } n_{i}^{\prime} \leqslant n \text {. }\end{array}$ \\
\hline$i$ & $\{i \mid 1 \leq i \leq m\}, m$ is the total number of small cells. \\
\hline$n_{i}^{\prime}-Y_{i}$ & $\begin{array}{l}\text { The remainder PRBs in the system resource pool } \\
\text { after pre-allocation for } s_{i} \text {. }\end{array}$ \\
\hline 1 & An interference matrix of small cells. \\
\hline$Y_{i}$ & The number of allocated PRBs in small cell $s_{i}$. \\
\hline \multirow[t]{2}{*}{$\mathcal{S}^{\prime}=\left\{s_{1}^{\prime}, \ldots, s_{m}^{\prime}\right\}$} & $\begin{array}{l}\text { The set of small cells in the whole network in } \\
\text { descending order based on the degree of vertex in }\end{array}$ \\
\hline & interference graph. \\
\hline $\mathcal{B S}_{i}^{\prime}$ & $\begin{array}{l}\text { The set of PRBs in small cell } s_{i} \text { after global pre- } \\
\text { allocation. }\end{array}$ \\
\hline $\mathcal{B}^{\prime}$ & $\begin{array}{l}\text { Total number of PRBs in the system resource pool } \\
\text { before resource optimization. }\end{array}$ \\
\hline $\mathcal{B}^{\prime \prime}$ & $\begin{array}{l}\text { Total number of PRBs in the system resource pool } \\
\text { after resource optimization. }\end{array}$ \\
\hline $\mathcal{B Z}_{s_{i}}$ & $\begin{array}{l}\text { The idle PRB list belonging to adjacent cells of small } \\
\text { cell } s_{i} \text {. }\end{array}$ \\
\hline $\mathcal{Z}_{s_{i}^{\prime \prime}}$ & The adjacent cell set of small cell $s_{i}^{\prime \prime}$. \\
\hline Odownstream & System downlink throughput. \\
\hline$O_{\text {downstream }}^{\prime}$ & $\begin{array}{l}\text { System downlink throughput after each resource } \\
\text { coordination. }\end{array}$ \\
\hline$t_{\text {delay }}$ & Average processing time caused by PRB switching. \\
\hline$V_{j}$ & The value of PRB $b_{j}$ \\
\hline $\mathcal{S}^{\prime \prime}=\left\{s_{1}^{\prime \prime}, s_{2}^{\prime \prime}, \ldots\right\}$ & The set of small cells with new PRB demands. \\
\hline $\mathcal{B S} \mathcal{S}_{\text {, }}^{\prime}$ & 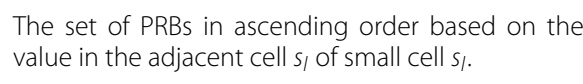 \\
\hline$O_{\text {downstream }}^{\prime}$ & $\begin{array}{l}\text { System downlink output after each resource coordi- } \\
\text { nation. }\end{array}$ \\
\hline$w_{j, l}$ & The order of PRB $b_{j}$ in $\mathcal{B S} \mathcal{S}_{/}^{\prime}$. \\
\hline 4 & The rate loss of the adjacent cell $s_{\text {l }}$ of small cell $s_{i}$. \\
\hline $\mathcal{T} \mathcal{S}_{l}$ & The set of transferred PRBs in small cell $s /$. \\
\hline
\end{tabular}

increases to above 210 and the overall trend becomes saturated, the algorithm is convergent. New users cannot access the network when there are more than 210 users. The throughput of the other two algorithms are lower than the proposed algorithm, and the proposed algorithm tended to be saturated more quickly; hence, the proposed algorithm clearly achieved the desired results of improving the system throughput.

Through the coordination of small cells, this algorithm demonstrated better performance compared to the other two algorithms for mitigating inter-cell interference and improving SINR. As illustrated in Fig. 7, when the number of users increased, the other two algorithms showed a sharp increase in interference, while the proposed algorithms shows a slow increase and its SINR tended to converge quickly.

\subsection{Analysis of user satisfaction}

User satisfaction is a comprehensive evaluation of users' successful access and continuous communication. The user is unable to access to the network until the small cell meets its rate demand, and the probability of successful access is $P_{\text {access }}$. With increasing user numbers, the PRB used in small cells will be transferred to the new users to ensure throughput maximization when the wireless resource is scarce, which will cause some communicating users of being dropped. The probability to be dropped is $P_{\text {drop. }}$

User satisfaction with the network services measures the network's performance. Here, the user satisfaction is defined as $P[28]$ :

$$
P=0.1 \times P_{\text {access }}+0.9 \times\left(1-P_{\text {drop }}\right) .
$$

From the user's experience with network services, the user prefers to be denied access rather than to be dropped. $1-P_{\text {drop }}$ stands for the probability of not being dropped; therefore, in this formula, the weight factor of successful access is 0.1 , and the weight factor of not being dropped is 0.9 .

As is shown in Fig. 8, with an increase in user numbers, user satisfaction starts to decline when the user number reaches 150 . User satisfaction decreases slowly and gradually trends plateaus when the user number is greater than 150. Compared to the other two algorithms, the proposed algorithm obtains a better user satisfaction. Because the algorithm solves the small cell resource allocation conflict harmonically in the local area, it reduces the PRB switching and users' outage probability.

\subsection{Analysis of signaling overheads}

Figure 9 offers a simple example to indicate that the proposed algorithm has less signaling than the other algorithms on the basis of two situations. 


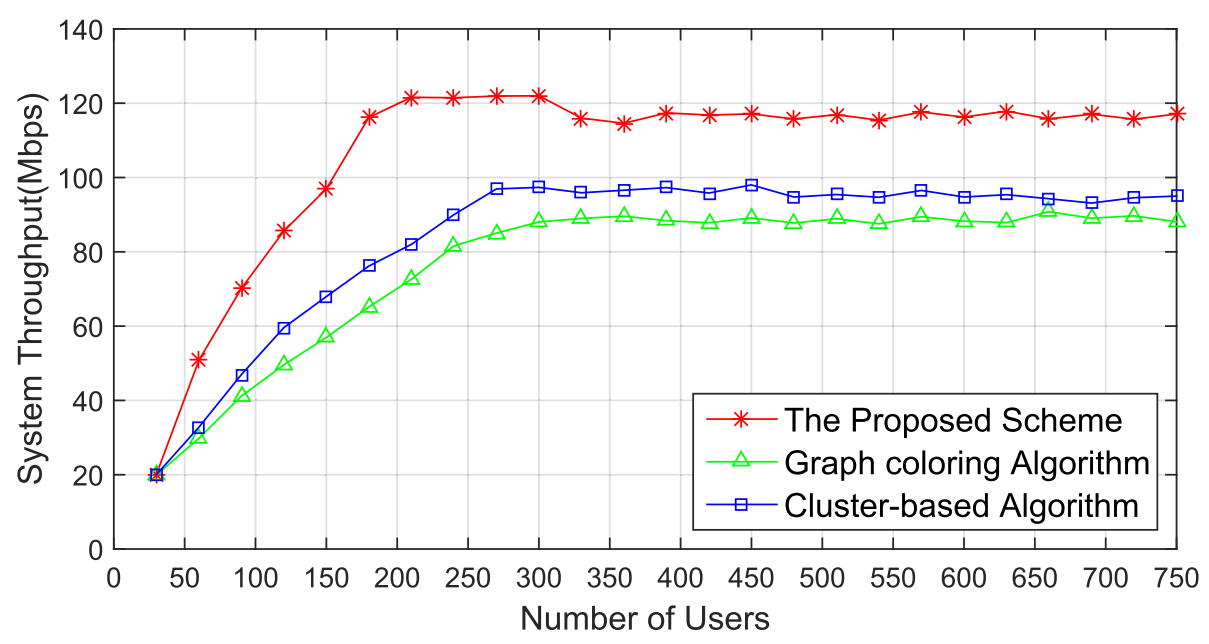

Fig. 6 Comparison of system throughput. Users include those with access and those denied access

In Fig. 9a, the PRB of the small cell is sufficient. In initial state, the "accessed UE" accesses the small cell, which needs two signaling (the solid line arrows). In general, the proposed algorithm causes less access delay for two signaling than the other two algorithms because the global resource allocation for the small cell managed by fog devices. When the "new UE" accesses the small cell, there are also two signaling between new UE and the small cell. The proposed algorithm has fewer instances of PRB switching than the other two algorithms. There are two extra signaling (the dotted arrows) in the other two algorithms because they require global resource allocation again once the new UE requests access, and the "accessed UE" in small cell may be assigned to a different PRB than before. In other words, the PRB switching is the main reason for the increase in signaling overhead.

In Fig. 9b, when the "new UE" requests to access the small cell $\beta$, the proposed algorithm will execute the resource coordination and scheduling process if the PRB of the small cell is deficient. If the small cell $\alpha$ has idle available PRB, it can borrow PRB to small cell $\beta$. Otherwise, the small cell can transfer the PRB with the smallest used value to the small cell $\beta$, which may lead to signaling for PRB switching; thus, there are two extra signaling between small cell $\alpha$ and the "accessed UE." The other two algorithms require the global resource allocation for all the UE within the network, so this process may cause two signaling to each "accessed UE."

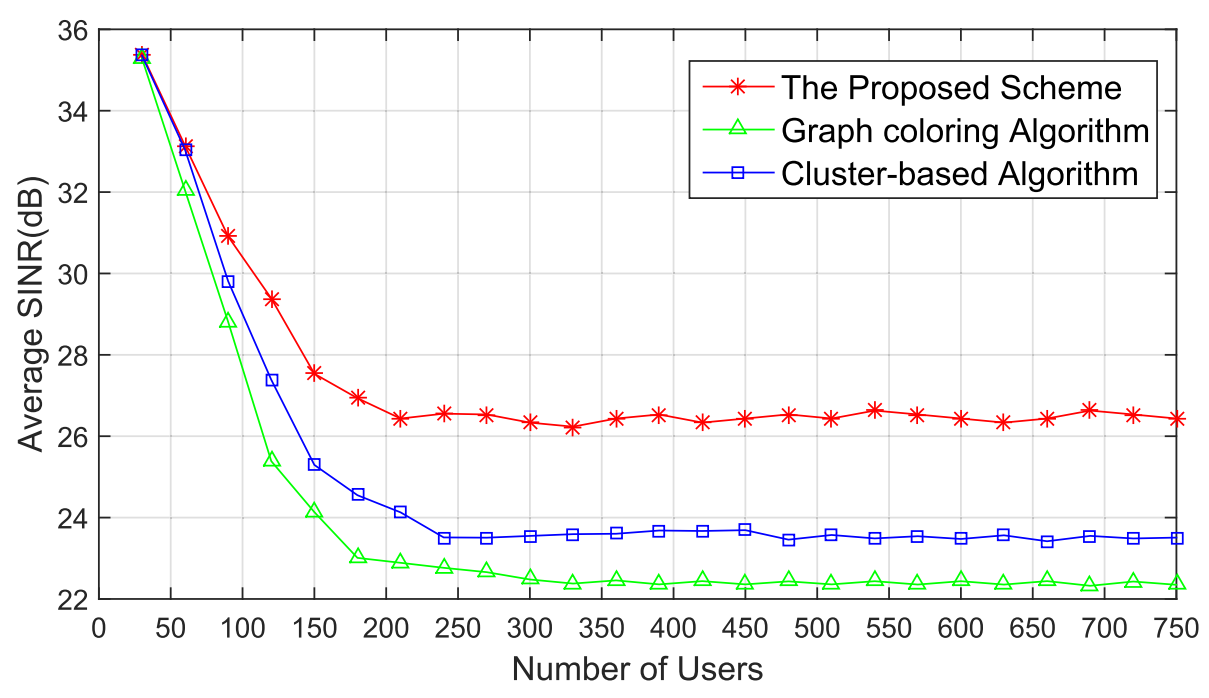

Fig. 7 Comparison of average SINR. Users include those with access and those denied access 


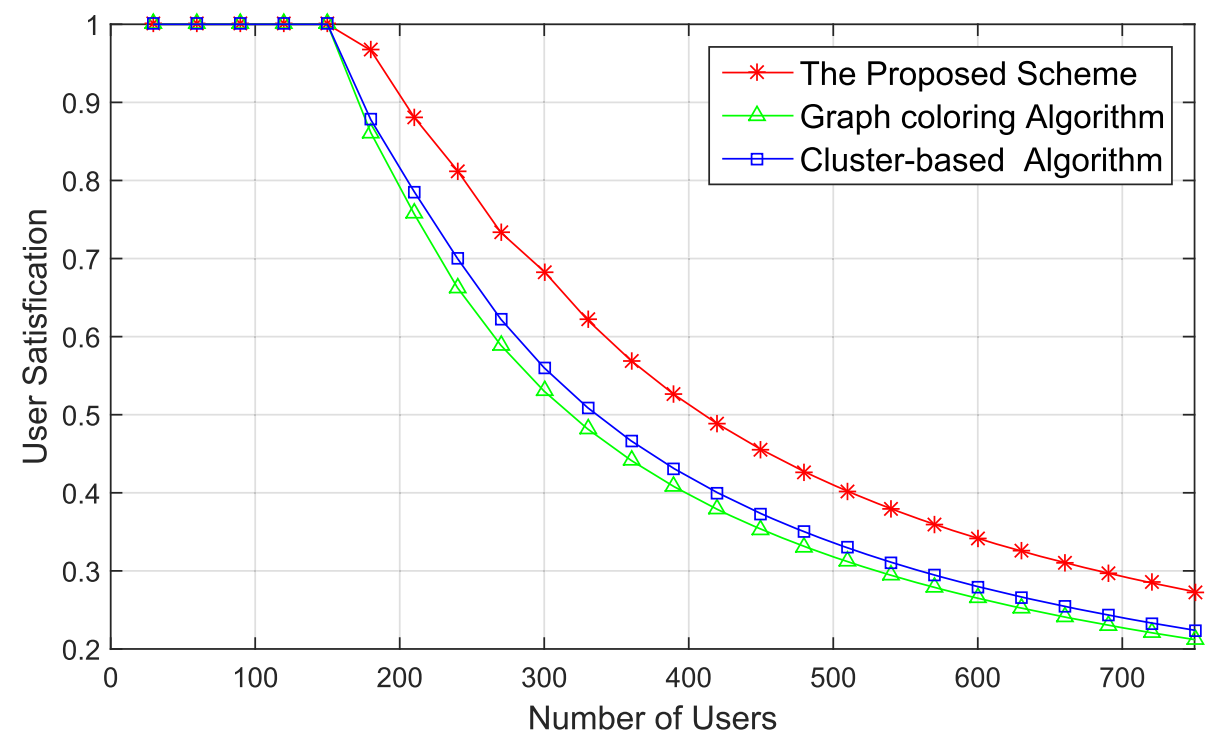

Fig. 8 Comparison of user satisfaction. Users include those with access and those denied access

Assume that the signaling overhead per PRB switch is equal to $20 \mathrm{~ms}$. As shown in Fig. 10, when the user number is less than 150 (i.e., the PRB is sufficient), the signaling overhead of the proposed algorithm remains at zero and the other two algorithms gradually increase as the user number increases. When the number of users is larger than 150 (i.e., the PRB is deficient), the signaling overhead of all three algorithms increases as the user number increases. When the user number reaches a certain value, the signaling overhead gradually decreases due to the number of access users becoming saturated, and the system capacity reaches its limit. Meanwhile, signaling overhead of the proposed algorithm is less than the other two algorithms, proving PRB switching times in this algorithm is less frequent than in the other two algorithms.

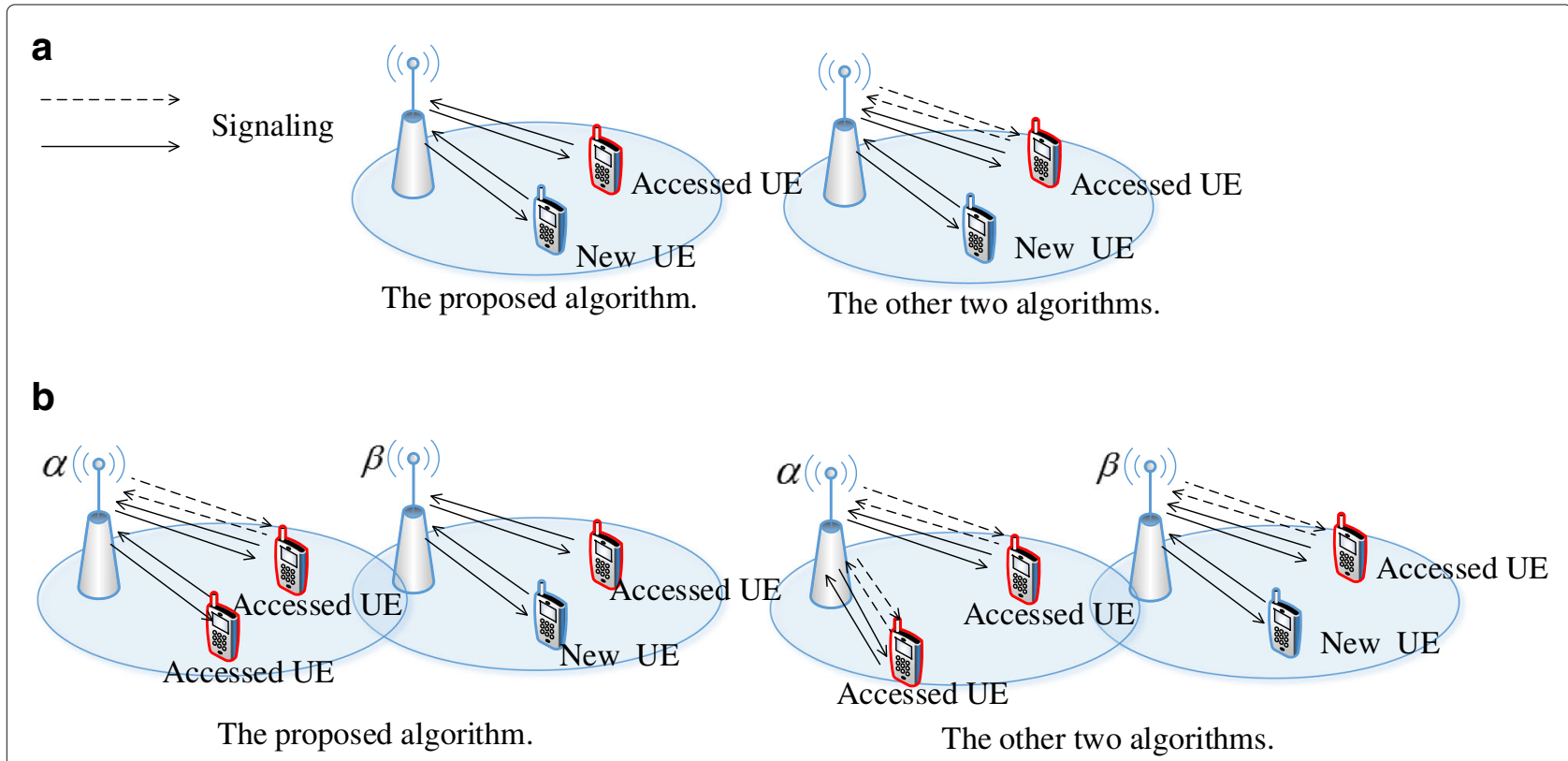

Fig. 9 Comparison of signaling in access process. $\mathbf{a}$ The PRB of small cell is sufficient. $\mathbf{b}$ The PRB of small cell is deficient 


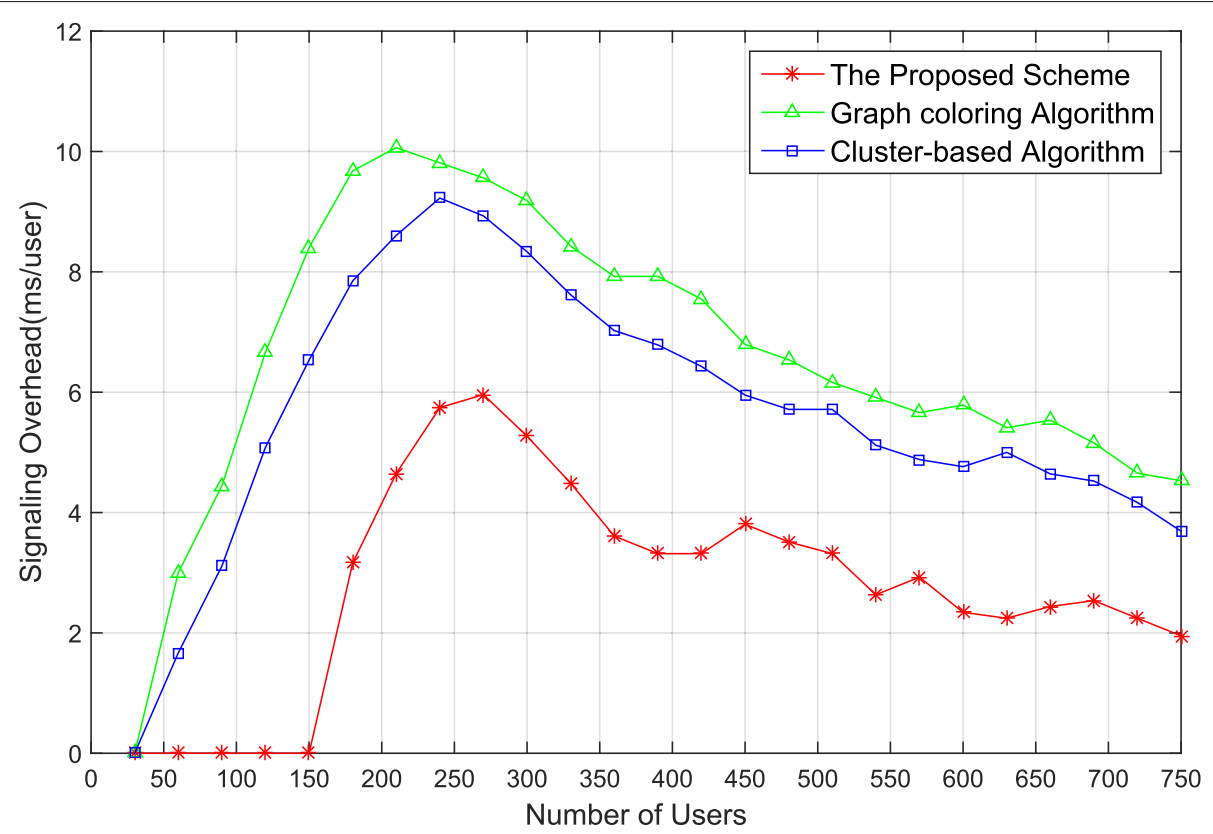

Fig. 10 Comparison of signaling overheads. Users include those with access and those denied access

\section{Conclusions}

The problems of interference and system overhead in ultra-dense small cells must be resolved. They depend on the efficient and rational allocation of radio resources. This study presents a novel radio resource coordination and scheduling scheme based on cloud infrastructure in an ultra-dense small cell network. In light of Cloud-RAN's improved computational ability, the global resource allocation and partial resource optimization with heavy computing tasks can be run on the cloud processing platform, considering the resource utilization and the fairness of resource allocation between small cells in the entire network. Meanwhile, because fog computing has a lower transmission delay, the resource coordination and scheduling algorithm are proposed for generally changeable small cells via the fog device, thereby avoiding excessive signaling overheads caused by using cloud computing. The simulation results show that the proposed scheme improves user satisfaction and downlink throughput and reduces system signaling overheads through the resource coordination of small cells.

\section{Acknowledgements}

This research was supported by the National Natural Science Foundation of China (Grant No. 61731012) and the National High Technology Research and Development Program of China (Grant No. 2015AA01A705). An earlier version of this paper was presented at the 2017 12th International Conference on Computer Science and Education (ICCSE). Special thanks are dedicated to Professor Weihua Zhuang's guidance, from the Department of Electrical and Computer Engineering at the University of Waterloo, Canada.

\section{Funding}

The research in this paper and its publication are supported by the National Natural Science Foundation of China (Grant No. 61731012) and the National High Technology Research and Development Program of China (Grant No. 2015AA01A705)

\section{Authors' contributions}

YT proposed the research ideas and directed the writing of the paper. ZC is the main author of this paper; she conducted the experiment and analyzed it with the help of SZ. Other authors promoted the quality of the entire manuscript. All authors read and approved the final manuscript.

\section{Competing interests}

The authors declare that they have no competing interests.

\section{Publisher's Note}

Springer Nature remains neutral with regard to jurisdictional claims in published maps and institutional affiliations.

\section{Author details}

${ }^{1}$ Department of Communication Engineering, Xiamen University, Xiamen 361005, China. ${ }^{2}$ Department of Computer and Information Sciences, Temple University, Philadelphia, USA. ${ }^{3}$ Department of Electrical and Computer Engineering, University of Idaho, Moscow, ID, USA.

Received: 31 March 2017 Accepted: 15 May 2018

Published online: 31 May 2018

\section{References}

1. S Chen, F Qin, B Hu, X Li, Z Chen, User-centric ultra-dense networks for $5 \mathrm{G}$ : challenges, methodologies, and directions. IEEE Wireless Commun. 23(2), 78-85 (2016)

2. O Semiari, W Saad, S Valentin, M Bennis, B Maham, in Acoustics, Speech and Signal Processing (ICASSP), 2014 IEEE International Conference On. Matching theory for priority-based cell association in the downlink of wireless small cell networks (IEEE, Florence, 2014), pp. 444-448

3. C Yang, J Li, M Guizani, Cooperation for spectral and energy efficiency in ultra-dense small cell networks. IEEE Wireless Commun. 23(1), 64-71 (2016) 
4. I Chih-Lin, J Huang, R Duan, C Cui, JX Jiang, L Li, Recent progress on C-RAN centralization and cloudification. IEEE Access. 2, 1030-1039 (2014)

5. JG Andrews, S Buzzi, W Choi, SV Hanly, A Lozano, AC Soong, JC Zhang, What will 5 G be? IEEE J. Selected Areas Commun. 32(6), 1065-1082 (2014)

6. S Zou, F Yang, Y Tang, L Xiao, The resource mapping algorithm of wireless virtualized networks for saving energy in ultradense small cells. Mob. Inf. Syst. 2015, 1-14 (2015)

7. S Sarkar, S Chatterjee, S Misra, Assessment of the suitability of fog computing in the context of internet of things. IEEE Trans. Cloud Comput. 6(1), 46-59 (2015)

8. M Bennis, SM Perlaza, P Blasco, Z Han, HV Poor, Self-organization in small cell networks: A reinforcement learning approach. IEEE Trans. Wireless Commun. 12(7), 3202-3212 (2013)

9. A Ghosh, R Ratasuk, B Mondal, N Mangalvedhe, T Thomas, LTE-advanced: next-generation wireless broadband technology [invited paper]. IEEE Wireless Commun. 17(3), 10-22 (2010)

10. S Bassoy, H Farooq, MA Imran, A Imran, Coordinated multi-point clustering schemes: a survey. IEEE Commun. Surv. Tutorials. 19(2), 743-763 (2017)

11. A Damnjanovic, J Montojo, Y Wei, T Ji, T Luo, M Vajapeyam, T Yoo, O Song, D Malladi, A survey on 3GPP heterogeneous networks. IEEE Wireless Commun. 18(3), 10-21 (2011)

12. Y Dong, Z Chen, P Fan, KB Letaief, Mobility-aware uplink interference model for $5 \mathrm{G}$ heterogeneous networks. IEEE Trans. Wireless Commun. 15(3), 2231-2244 (2016)

13. J Zheng, Y Cai, A Anpalagan, A stochastic game-theoretic approach for interference mitigation in small cell networks. IEEE Commun. Lett. 19(2), 251-254 (2015)

14. S Lin, H Tian, in Wireless Communications and Networking Conference (WCNC), 2013 IEEE. Clustering based interference management for QoS guarantees in OFDMA femtocell (IEEE, Shanghai, 2013), pp. 649-654

15. Y Zhang, S Wang, J Guo, in Communications in China (ICCC), 2015 IEEE/CIC International Conference On. Clustering-based interference management in densely deployed femtocell networks (EEE, Shenzhen, 2015), pp. 1-6

16. A Hatoum, R Langar, N Aitsaadi, R Boutaba, G Pujolle, Cluster-based resource management in OFDMA femtocell networks with QoS guarantees. IEEE Trans. Veh. Technol. 63(5), 2378-2391 (2014)

17. A Abdelnasser, E Hossain, DI Kim, Clustering and resource allocation for dense femtocells in a two-tier cellular OFDMA network. IEEE Trans. Wireless Commun. 13(3), 1628-1641 (2014)

18. T Lotfollahzadeh, S Kabiri, H Kalbkhani, MG Shayesteh, Femtocell base station clustering and logistic smooth transition autoregressive-based predicted signal-to-interference-plus-noise ratio for performance improvement of two-tier macro/femtocell networks. IET Signal Process. 10(1), 1-11 (2016)

19. Z Sun, R Liu, W Wang, Joint time-frequency domain cyclostationaritybased approach to blind estimation of OFDM transmission parameters. EURASIP J. Wireless Commun. Netw. 2013(1), 1-8 (2013)

20. B Han, W Wang, Y Li, M Peng, Investigation of interference margin for the co-existence of macrocell and femtocell in orthogonal frequency division multiple access systems. IEEE Syst. J. 7(1), 59-67 (2013)

21. TA Weiss, FK Jondral, Spectrum pooling: an innovative strategy for the enhancement of spectrum efficiency. IEEE Commun. Mag. 42(3), 8-14 (2004)

22. J Holdren, E Lander, Realizing the full potential of government-held spectrum to spur economic growth. Tech. Rep. (2012)

23. Y Li, Z Feng, S Chen, Y Chen, D Xu, P Zhang, Q Zhang, Radio resource management for public femtocell networks. EURASIP J. Wireless Commun. Netw. 2011(1), 1-16 (2011)

24. Y Li, Z Feng, D Xu, Q Zhang, H Tian, Optimisation approach for femtocell networks using coordinated multipoint transmission technique. Electron. Lett. 47(24), 1348-1349 (2011)

25. Z Chen, Y Tang, in Computer Science and Education (ICCSE), 2017 12th International Conference On. A resource collaboration scheduling scheme in ultra-dense small cells (IEEE, Houston, 2017), pp. 401-405

26. H Aissi, C Bazgan, D Vanderpooten, Complexity of the min-max and min-max regret assignment problems. Oper. Res. Lett. 33(6), 634-640 (2005)

27. A Mehrotra, MA Trick, A branch-and-price approach for graph multi-coloring. Oper. Res. Comput. Sci. Interfaces. 37, 15-29 (2010)

28. Y Wang, P Zhang, Radio resource management. (Beijing University of Posts and Telecommunications Press, China, 2005), p. 21

\section{Submit your manuscript to a SpringerOpen ${ }^{\circ}$ journal and benefit from:}

- Convenient online submission

- Rigorous peer review

- Open access: articles freely available online

- High visibility within the field

- Retaining the copyright to your article

Submit your next manuscript at $\boldsymbol{~ s p r i n g e r o p e n . c o m ~}$ 